\title{
TOWARDS A PHILOSOPHICAL UNDERSTANDING OF THE LOGICS OF FORMAL INCONSISTENCY
}

\author{
WALTER CARNIELLI ${ }^{1}$, ABÍLIO RODRIGUES ${ }^{2}$
}

CLE and Department of Philosophy

State University of Campinas, UNICAMP

CAMPINAS, SP

BRAZIL

walter.carnielli@cle.unicamp.br

${ }^{2}$ Department of Philosophy

Federal University of Minas Gerais, UFMG

BELO HORIZONTE, $M G$

$B R A Z I L$

abilio@ufmg.br

\section{Received: 13.12.2014; Accepted: 08.07.2015}

\begin{abstract}
In this paper we present a philosophical motivation for the logics of formal inconsistency, a family of paraconsistent logics whose distinctive feature is that of having resources for expressing the notion of consistency within the object language in such a way that consistency may be logically independent of non-contradiction. We defend the view according to which logics of formal inconsistency may be interpreted as theories of logical consequence of an epistemological character. We also argue that in order to philosophically justify paraconsistency there is no need to endorse dialetheism, the thesis that there are true contradictions. Furthermore, we show that $m b C$, a logic of formal inconsistency based on classical logic, may be enhanced in order to express the basic ideas of an intuitive interpretation of contradictions as conflicting evidence.
\end{abstract}

Keywords: paraconsistent logics, logics of formal inconsistency, philosophy of paraconsistency, non-classical logics

The aim of this paper is twofold. Firstly, we want to present a philosophical motivation for the logics of formal inconsistency (LFIs), a family of paraconsistent logics whose distinctive feature is that of having

Manuscrito - Rev. Int. Fil., Campinas, v. 38, n.2, pp.155-184, jul.-ago. 2015. 
resources for expressing the notion of consistency within the object language in such a way that consistency may be logically independent of non-contradiction. We shall defend the view according to which logics of formal inconsistency may be interpreted as theories of logical consequence of a normative and epistemological character. We will see that in order to justify paraconsistency from the philosophical viewpoint there is no need to endorse dialetheism, the thesis that there are true contradictions.

Secondly, with respect to $m b C$, an LFI based on classical logic presented in Carnielli et al. (2007) ${ }^{1}$, we show how its syntax and semantics can be enhanced in order to express the basic features of the following intuitive reading for the paraconsistent negation: the acceptance of $\neg A$ means that there is some evidence that $A$ is not the case. If such evidence is non-conclusive, it may be that there is simultaneously some evidence that $A$ is the case. A contradiction is therefore interpreted not as the simultaneous truth of $A$ and $\neg A$, but rather as simultaneous evidence for truth and falsity of $A$. Conclusive evidence is tantamount to truth, and if there is conclusive evidence for $A$, it cancels any evidence for $\neg A$ (mutatis mutandis for $\neg A$ and $A$ ). Therefore, the acceptance of a pair of contradictory propositions $A$ and $\neg A$ need not to be taken in the strong sense that both are true. ${ }^{2}$

In section 1 , in order to give a general view of the problem dealt with by paraconsistent logics, namely, that of dealing with contexts of reasoning in which contradictions occur, we shall present the distinction between explosiveness and contradictoriness. This distinction is essential for grasping the distinction between classical and paraconsistent logics. In section 2, we explain what we mean by 'epistemological contradictions' and argue that at least some contradictions that appear in

1 The name ' $m b C$ ' stands for 'a minimal logic with the axiom bcl', and 'bc' stands intuitively for 'basic property of consistency'.

${ }^{2}$ The symbol ' $\neg$ ' will be used here as a paraconsistent negation, in contrast to classical negation ' $\sim$ '.

Manuscrito - Rev. Int. Fil., Campinas, v. 38, n.2, pp.155-184, jul.-ago. 2015. 
empirical sciences have an epistemological character. In section 3, we present an axiomatic system for $m b C$ with a correct and complete semantics and a slightly modified system called $m b C D$ that is not only paraconsistent but also paracomplete. In this way, $m b C D$ is made suitable to the intuitive interpretation of contradictions as conflicting evidence. In section 4, we shall examine the problem of the nature of logic, namely, whether logic as a theory of logical consequence has primarily an ontological or an epistemological character. The point is not to give a definite answer to this problem but, rather, to clarify and understand important aspects of paraconsistent logic. We defend the view that logics of formal inconsistency are well suited to the epistemological side of logic and fit an intuitive justification of paraconsistency that is not committed to dialetheism. ${ }^{3}$

\section{CONTRADICTIONS AND EXPLOSIONS}

Classical logic does not accept contradictions. This is not only because it endorses the validity of the principle of non-contradiction, $\sim(A \wedge \sim A)$, but more importantly because, classically, everything follows from a contradiction. This is the inference rule called ex falso quodlibet, or law of explosion,

(1) $A, \sim A \vdash B$.

From a pair of propositions $A$ and $\sim A$, we can prove any proposition through logic, from ' $2+2=5$ ' to 'snow is black'.

\footnotetext{
${ }^{3}$ One of the intentions of this paper is to present the logics of formal inconsistency to the non-technical minded reader. We presuppose only a basic knowledge of classical logic. The paper, we hope, is as intuitive as it can be within the space allowed, while at the same time dealing with aspects of logics of formal inconsistency that are yet unexplored.
}

Manuscrito - Rev. Int. Fil., Campinas, v. 38, n.2, pp.155-184, jul.-ago. 2015. 
Before the rise of modern logic (i.e., that which emerged in the late nineteenth century with the works of Boole and Frege), the validity of the principle of explosion was still a somewhat contentious issue. However, in Frege's Begriffsschrift, where we find for the first time a complete system of what later would be called first-order logic, the principle of explosion holds (proposition 36 of part II). It is worth noting in passing that Frege's logicist project failed precisely because his system was inconsistent, and therefore trivial. Frege strongly attacked the influence of psychologism in logic (we will return to this point later), and in his writings we find a realist conception of mathematics and logic that contributed to emphasizing the ontological and realist vein of classical logic. It is true that one may endorse classical logic without being a realist. However, the unrestricted validity of excluded middle and noncontradiction, an essential feature of classical logic, is perfectly suited to the realist view according to which reality ultimately decides the truth value of every meaningful proposition independently of our ability to know it.

The account of logical consequence that was established as standard in the first half of twentieth century through the works of Russell, Tarski, and Quine, among others, is classical. Classical logic is invariably the logic we first study in introductory logic books, and the law of explosion holds in it, where consistency is tantamount to freedom from contradiction.

Let us put these things a bit more precisely. Let $T$ be a theory formulated in some language, call it $L$, whose underlying logic is classical. If $T$ proves a pair of propositions $A$ and $\sim A, \sim$ being classical negation, then $T$ proves all of the propositions of $L$. In this case, we say that $T$ is trivial. For this reason, contradictions must be avoided at all costs in classical logic. The most serious consequence of a contradiction in a classical theory is not the violation of the principle of non-contradiction, but rather the trivialization of the theory. The central point of paraconsistency is that these two things, contradiction and triviality, do not need to be the same thing. 
Although classical logic has become the standard approach, several alternative accounts of logical consequence have been proposed and studied. At the beginning of the twentieth century, Brouwer (1907), motivated by considerations regarding the nature of mathematical knowledge, established the basis for intuitionistic logic, a different account of logical consequence. The principle of excluded middle, a socalled 'fundamental law of thought', together with the principle of noncontradiction, is not valid in intuitionistic logic.

Intuitionistic logic, later formalized by Heyting (1956), a former student of Brouwer, has an epistemological character in clear opposition to Frege's realism. In brief, starting from the assumption that mathematical objects are not discovered but rather created by the human mind, the intuitionists' motivation for rejecting the excluded middle is the existence of mathematical problems for which there are no known solutions. The usual example is the Goldbach conjecture: every even number greater than 2 is the sum of two prime numbers. Let us call this proposition $G$. Until now, there is no proof of $G$, nor is there a counterexample of an even number greater than 2 that is not the sum of two primes. The latter would be a proof of not $G$. For this reason, the intuitionist maintains that we cannot assert the relevant instance of excluded middle, $G$ or not $G$. Doing so would commit us to a Platonic, supersensible realm of previously given mathematical objects.

So-called classical logic is based on principles that, when rejected, may give rise to alternative accounts of logical consequence. Two accepted principles of classical logic are the aforementioned laws of excluded middle and non-contradiction. The principle of noncontradiction can be interpreted from an ontological point of view as a principle about reality, according to which facts, events, mathematical objects, etc., cannot be in contradiction with each other. Its formulation in first order logic,

$$
\forall x \sim(P x \wedge \sim P x),
$$

says that it cannot be the case that an object simultaneously has and does not have a given property $P$. Note (and this is a point we want to emphasize) that the principle of explosion can be understood as a still 
more incisive way of saying that there can be no contradiction in reality otherwise everything is the case, and we know this cannot be so. Explosion is thus a stronger way of expressing an idea usually attributed to non-contradiction. In paraconsistent logics the law of explosion is not valid. Thus, the central question for an intuitive interpretation for paraconsistency is the following:

What does it mean to accept a pair of contradictory propositions $A$ and $\neg A$ ?

\section{ON EPISTEMOLOGICAL CONTRADICTIONS}

Contradictions appear in a number of contexts of reasoning, among which three are worth mentioning: (i) computational databases; (ii) semantic and set theoretic paradoxes; (iii) scientific theories. In the first case, contradictions invariably have a provisional character, as an indication of an error to be corrected. We will make only few remarks with regard to the second case, because an analysis of this problem requires a technical exposition that cannot be gone into here. The third case will be discussed below.

Naive set theory, which yields set theoretic paradoxes, has been revised and corrected. Up to the present time, there is no indication that ZFC (and its variants) is not consistent - in fact, all indications are to the contrary. The moral we can draw from this is that our intuitive conception of set is defective, a 'product of thought', so to speak, that yields contradictions. Nothing more should be concluded. With respect to semantic paradoxes, they are results about languages with certain characteristics. It is worth noting that the diagonal lemma, essential in the formalization of both the Liar and Curry's paradoxes, is a result about language itself. Thus we believe that the step from paradoxes to the claim that there are true contradictions, in the sense that reality is contradictory, should not be taken. 
Here we will concentrate on the third case, the occurrence of contradictions in scientific theories. Although mathematicians do their work based on the assumption that mathematics is free of contradictions, in empirical sciences contradictions seem to be unavoidable, and the presence of contradictions is not a sufficient condition for throwing interesting theories away. According to da Costa and French (2003, p. 84), "the prevalence of inconsistency or of 'incongruent' suppositions [...] in both our scientific and 'everyday' belief structures is something that is being increasingly recognized", and they explain "incongruent suppositions" as "those aspects of scientific and everyday belief structures that, when rendered less vague and more precise, can be represented as inconsistencies" (p. 216). da Costa and French (p. 84ff) also mention several examples of scientific theories that were inconsistent in themselves or yielded inconsistencies when put together with other theories. Notice that, as a general rule, these theories have been successful in describing and predicting a wide range of phenomena. The realist (and naive) assumption that scientific theories provide correct descriptions of reality would unavoidably imply dialetheism. But this would be a careless and hasty conclusion, since these inconsistencies in a number of circumstances are better taken as provisional. This situation is well illustrated by the following passage from Nickles (2002, p. 2):

Methodologists of science now appreciate more than ever that the
empirical sciences $[\ldots]$ are not monotonic, foundational disciplines but
rather nonmonotonic enterprises in which well justified results are
routinely overturned or seriously qualified by later results. And
'nonmonotonic' implies 'temporally inconsistent' if we suppose
(plausibly) that the results become known successively in time and are
logically detachable from their evidence bases.

If inconsistencies, or rather contradictions, are provisional, they should not be taken as true contradictions. From a strictly logical point of view, the problem we have on our hands is how to formulate an account of logical consequence capable of identifying, in such contexts, the 
inferences that are allowed, distinguishing them from those that must be blocked. It is clear that such an account of logical consequence must be paraconsistent. However, from a philosophical point of view, a question poses itself: what can be said about the nature of these contradictions? We are not going to discuss whether or not some of these contradictions indeed belong to reality, and thus are not provisional, because it is a problem that cannot be solved by philosophy alone. But we can safely say that at least some of these contradictions have their origin in our cognitive apparatus, in the failure of measuring instruments, in the interactions of these instruments with phenomena, or even in simple mistakes that can be corrected later on. Such contradictions are what we call epistemological contradictions.

\section{FROM $m b c$ TO $m b C D$ : TWO MINIMAL LOGICS OF FORMAL INCONSISTENCY}

To begin, let us recall some useful definitions. A theory is a set of propositions closed under logical consequence. This means that everything that is a logical consequence of the theory is also part of the theory. For instance, everything that is a consequence of the basic principles of arithmetic, for instance, ' $5+7=12$ ', is also part of arithmetic. We say that a theory $T$ is:

(i) contradictory if and only if there is a proposition $A$ in the language of $T$ such that $T$ proves $A$ and $\sim A$ (i.e., $T$ proves a contradiction);

(ii) trivial if and only if for any proposition $A$ in the language of $T, T$ proves $A$ (i.e., $T$ proves everything);

(iii) explosive if and only if $T$ is trivialized when exposed to a pair of contradictory formulas (i.e., for all $A$ and $B, T \cup\{A, \sim A\} \vdash B$ ).

There is a difference between a theory being contradictory and being trivial. Contradictoriness entails triviality only when the principle of explosion holds. Without explosion, we may have contradictions without triviality. 
In books on logic, we find two different but classically equivalent notions of consistency. Hunter (1973, p. 78ff) calls them simple and absolute consistency. A deductive system $S$ with a negation $\sim$ is simply consistent if and only if there is no formula $A$ such that $\vdash S A$ and $\vdash S$ $\sim A$. The other notion of consistency, absolute consistency, says that a system $S$ is consistent if and only if there is a formula $B$ such that $\forall_{S} B$. In other words, $S$ does not prove everything. The latter notion is tantamount to saying that $S$ is not trivial, while the former is tantamount to saying that $S$ is non-contradictory. From the point of view of classical logic, both notions are equivalent because the principle of explosion holds. The proof is simple and easy. Suppose $S$ is trivial. Hence, it proves everything, including a pair of propositions $A$ and $\sim A$. Now suppose $S$ is contradictory, that is, it proves a pair of propositions $A$ and $\sim A$. If the principle of explosion holds in $S$, as is the case in classical logic, then $S$ proves everything, hence $S$ is trivial. Paraconsistent logics separate triviality from inconsistency, restricting the principle of explosion.

In logics of formal inconsistency the principle of explosion is not valid in general, that is, it is not the case that from any pair of contradictory propositions everything follows. These logics contain a non-explosive negation, represented here by “ $\neg$ ", such that for some $A$ and $B$,

$$
A, \neg A \nvdash B \text {. }
$$

In addition, there is a unary consistency connective called 'ball': ' $\circ A$ ' means informally that $A$ is consistent. We can thus isolate contradictions in such a way that the application of the law of explosion is restricted to consistent propositions only, thus avoiding triviality even in the presence of one or more contradictions. Therefore, a contradictory theory may be non-trivial. However, as we will see, we can go further and separate the concept of inconsistency from that of contradictoriness. Consistency may be taken as a primitive notion, its meaning being elucidated from outside the formal system. We will return to this point later on. 
It is worth mentioning here a common misunderstanding about paraconsistent logics in general. It is true that in the majority of paraconsistent logics the principle of non-contradiction is not valid. However, this does not mean that deductive systems of paraconsistent logic have contradictions as theorems. This is a mistake similar to saying that intuitionistic logic proves the negation of excluded middle.

The fundamental distinction between classical logic and paraconsistent logics occurs at the sentential level. In what follows, we will start by presenting an axiomatic system of $m b C$, a sentential logic of formal inconsistency presented for the first time in Carnielli and Marcos (2002) and studied in detail in Carnieli et al. (2007). We show how a few modifications on $m b C$ define the paraconsistent (and paracomplete) logic $m b C D$.

Let $L$ be a language with sentential letters, the set of logical connectives $\{\vee, \wedge, \rightarrow, \neg, \circ\}$, and parentheses. Notice that the consistency operator ' $\circ$ ', mentioned above, is a primitive symbol. The set of formulas of $L$ is obtained recursively in the usual way. Consider the following axiom-schemas:

Ax. 1. $\mathrm{A} \rightarrow(\mathrm{B} \rightarrow \mathrm{A})$

Ax. 2. $(\mathrm{A} \rightarrow(\mathrm{B} \rightarrow \mathrm{C})) \rightarrow((\mathrm{A} \rightarrow \mathrm{B}) \rightarrow(\mathrm{A} \rightarrow \mathrm{C}))$

Ax. 3. $\mathrm{A} \rightarrow(\mathrm{B} \rightarrow(\mathrm{A} \mathrm{B}))$

Ax. 4. $(\mathrm{A} \wedge \mathrm{B}) \rightarrow \mathrm{A}$

Ax. 5. $(\mathrm{A} \wedge \mathrm{B}) \rightarrow \mathrm{B}$

Ax. 6. $A \rightarrow(A \vee B)$

Ax. 7. $\mathrm{B} \rightarrow(\mathrm{A} \vee \mathrm{B})$

Ax. 8. $(\mathrm{A} \rightarrow \mathrm{C}) \rightarrow((\mathrm{B} \rightarrow \mathrm{C}) \rightarrow((\mathrm{A} \vee \mathrm{B}) \rightarrow \mathrm{C}))$

Ax. 9. $A \vee(A \rightarrow B)$

Ax. 10. $A \vee \neg A$

Ax. bc1. $\circ \mathrm{A} \rightarrow(\mathrm{A} \rightarrow(\neg \mathrm{A} \rightarrow \mathrm{B}))$

Inference rule: modus ponens

The definition of a proof of $A$ from premises $\Gamma(\Gamma \vdash \mathrm{mbC} A)$ is the usual one: a sequence of formulas $B_{1} \ldots B_{n}$ such that $A$ is $B_{n}$ and each 
$B_{i}(1 \leq i \leq n)$ is an axiom, a formula that belongs to $\Gamma$, or the result of modus ponens. ${ }^{4}$ Thus, monotonicity holds:

(2) if $\Gamma \vdash B$, then $\Gamma, A \vdash B$, for any $A$.

A theorem is a formula proved from the empty set of premises. In $m b C, A x .1$ and Ax.2, plus modus ponens grant the Deduction Theorem:

(3) if $\Gamma, A \vdash B$, then $\Gamma \vdash A \rightarrow B$.

We thus have inferences that correspond to introduction and elimination rules of implication in a natural deduction system. Since monotonicity holds, we also have the converse of the Deduction Theorem. Hence,

(4) $\Gamma, A \vdash B$ if and only if $\Gamma \vdash A \rightarrow B$.

Axioms 3-5 and 6-8 correspond, respectively, to the introduction and elimination rules of conjunction and disjunction in a natural deduction system. Axioms 1-9 form a complete system of positive classical logic, i.e., a system that proves all classical tautologies that can be formed with $\rightarrow, \vee$ and $\wedge$. Negation shows up in Ax.10 and Ax.11. Excluded middle for paraconsistent negation holds (more on this point soon), and explosion is restricted to consistent formulas. Consistency is a primitive notion; it is not definable in terms of non-contradiction, that is, $\neg(A \wedge \neg A)$ and $\circ A$ are not equivalent. It is clear that in a system whose consequence relation $\vdash$ enjoys the deduction theorem in both directions, the law of explosion

$$
\text { (1) } \mathrm{A}, \neg \mathrm{A} \vdash \mathrm{B}
$$

and the axiom schema

$$
(5) \vdash \mathrm{A} \rightarrow(\neg \mathrm{A} \rightarrow \mathrm{B})
$$
explosion.

are equivalent. To make things simpler, we call both the law of

The system is not explosive with respect to non-consistent formulas. For this reason, we say that it is gently explosive. If we simultaneously have contradiction and consistency, the system explodes, becoming trivial.

\footnotetext{
${ }^{4}$ When there is no risk of ambiguity, we drop the subscripts and just write $\vDash$ and $\vdash$.
}

Manuscrito - Rev. Int. Fil., Campinas, v. 38, n.2, pp.155-184, jul.-ago. 2015. 
The point, however, is precisely this: there cannot be consistency and contradiction, simultaneously and with respect to the same formula. If explosion in classical logic means that there can be no contradiction at all, the restricted principle of explosion may be understood as a more refined way of stating the same basic idea: there can be no contradiction with respect to consistent propositions.

A remarkable feature of $m b C$ is that it can be seen as an extension of classical logic. We can define a bottom particle as follows:

(6) $\perp \stackrel{\text { def }}{=} \circ \mathrm{A} \wedge \mathrm{A} \wedge \neg \mathrm{A}$.

Now, as an instance of Ax.9, we get

(7) $\mathrm{A} \vee(\mathrm{A} \rightarrow \perp)$.

We also have that

(8) $\vdash \perp \rightarrow B$,

since, by Ax.bc1,

(9) $\circ \mathrm{A} \wedge \mathrm{A} \wedge \neg \mathrm{A} \vdash \mathrm{B}$.

Axioms 1-8 plus (7) and (8) give us classical logic. ${ }^{5}$

A point that has certainly already occurred to the reader regards the interpretation of the system above. Three questions that pose themselves are:

(i) How can a semantics be given for the formal system above?

(ii) What is the intuitive meaning of the consistency operator?

(iii) How can an intuitive interpretation for contradictory formulas be provided?

In Carnielli et al. (2007, pp. 38ff), we find a bivalued non-truthfunctional semantics that is complete and correct for $m b C$. An $m b C$ valuation is a function that attributes values 0 and 1 to formulas of $L$, satisfying the following clauses:

(i) $\mathrm{v}(\mathrm{A} \wedge \mathrm{B})=1$ if and only if $\mathrm{v}(\mathrm{A})=1$ and $\mathrm{v}(\mathrm{B})=1$

(ii) $\mathrm{v}(\mathrm{A} \vee \mathrm{B})=1$ if and only if $\mathrm{v}(\mathrm{A})=1$ or $\mathrm{v}(\mathrm{B})=1$

${ }^{5}$ A complete axiomatization for classical sentential logic, in a language in which $\sim \mathrm{A}$ is defined as $\mathrm{A} \rightarrow \perp$, is given by axioms 1-8 plus $(*) \sim \sim \mathrm{A} \rightarrow \mathrm{A}$ (see Robbin 1997, chapter $1)$. We leave it as an exercise to the reader to prove $(*)$ in the system given by axioms 1 8 plus (7) and (8).

Manuscrito - Rev. Int. Fil., Campinas, v. 38, n.2, pp.155-184, jul.-ago. 2015. 
(iii) $\mathrm{v}(\mathrm{A} \rightarrow \mathrm{B})=1$ if and only if $\mathrm{v}(\mathrm{A})=0$ or $\mathrm{v}(\mathrm{B})=1$

(iv) $\mathrm{v}(\neg \mathrm{A})=0$ implies $\mathrm{v}(\mathrm{A})=1$

(v) $\mathrm{v}(\circ \mathrm{A})=1$ implies $\mathrm{v}(\mathrm{A})=0$ or $\mathrm{v}(\neg \mathrm{A})=0$

A valuation $v$ is a model of $\Gamma$ if and only if every proposition of $\Gamma$ receives the value 1 in $\nu$. The notion of logical consequence is defined as usual: $\Gamma \vDash_{\mathrm{mbC}} A$ if and only if for every valuation $v$, if $v$ is a model of $\Gamma, v(A)=1$.

We suggest that the values 0 and 1 (attributed to formulas without the occurrence of $\circ$ ) should not be understood as false and true simpliciter, but rather as expressing the existence of evidence:

$v(A)=1$ means that there is evidence that $A$ is the case;

$v(A)=0$ means that there is no evidence that $A$ is the case;

$v(\neg A)=1$ means that there is evidence that $A$ is not the case;

$v(\neg A)=0$ means that there is no evidence that $A$ is not the case.

Notice that 'there is no evidence that $A$ is not the case' means the same as 'there is no evidence for $\neg A$ '. Both are represented in the semantics by $v(\neg A)=0$. Similarly, 'there is evidence that $A$ is not the case' means the same as 'there is evidence for $\neg A$ ', both represented by $v(\neg A)=1$. There is no distinction between 'evidence for $\neg A$ ' and 'evidence that $A$ is not the case', nor between 'evidence for $A$ ' and 'evidence that $A$ is the case'.

The following passage from da Costa (1982 pp. 9-10) helps to elucidate what we mean by evidence.

Let us suppose that we want to define an operational concept of negation, at least for the negation of some atomic sentences. $\neg \mathrm{A}$, where $\mathrm{A}$ is atomic, is to be true if, and only if, the clauses of an appropriate criterion $c$ are fulfilled, clauses that must be empirically testable; i.e., we have an empirical criterion for the truth of the negation of A. Naturally, the same must be valid for the atomic proposition $A$, for the sake of coherence. Hence, there exists a criterion $d$ for the truth of A. But clearly it may happen that the criteria $c$ and $d$ be such that they entail, under certain critical circumstances, the truth of both $\mathrm{A}$ and $\neg \mathrm{A}$.

Although da Costa in the passage above is talking about a criterion of truth, it seems to us that it is much more reasonable, in a situation such as the one there described, not to draw the conclusion 
that $A$ and $\neg A$ are both true. It is better to be more careful and to take the contradictory data only as a sort of a provisional state. Accordingly, the criteria $c$ and $d$ constitute reasons to believe that respectively $\neg A$ and $A$ are true, but they do not establish conclusively that both are true

Now we turn to formulas of the kind $\circ A$ and suggest the following intuitive interpretation: established.

$v(\circ \mathrm{A})=1$ means that the truth value of $A$ has been conclusively

Accordingly, $\circ A$ says something about the justification of $A$, or of $\neg A$, but what exactly such a justification would be is not a problem of logic. It is the physicist, the chemist, the mathematician, etc., who is able, with respect to concrete situations, to say what constitutes a conclusive establishment of the truth of a proposition $A$. It is important here to call attention to the fact that what constitutes evidence for a given proposition $A$, and whether or not such evidence is conclusive and $A$ may be established as true, are problems that depend on the specific area of knowledge being dealt with. These problems are external to the formal system. It is the user of the system who establishes, in accordance to specific criteria, that such and such formulas may be 'marked' with $\circ$.

Note that, since excluded middle holds in $m b C$ (axiom 10), a scenario is ruled out such that there is no evidence at all, either for $A$ or for $\neg A$. Indeed, a valuation $v$ such that $v(\mathrm{~A})=0$ and $v(\neg \mathrm{A})=0$ is forbidden by clause (iv) and would invalidate excluded middle. However, some small modifications may be easily implemented in $m b C$. We drop axiom 10 , substituting it by

$(\mathrm{bd} 1) \circ \mathrm{A} \rightarrow(\mathrm{A} \vee \neg \mathrm{A})$.

We may understand $b d 1$ as saying that if $A$ is determined, then excluded middle holds with respect to $A$ '. In order to get a complete and correct semantics, we drop clauses (iv) and (v), substituting them by the clause

(vi) $\mathrm{v}(\circ \mathrm{A})=1$ implies $[\mathrm{v}(\mathrm{A})=0$ iff $\mathrm{v}(\neg \mathrm{A})=1]$.

The logic so obtained we call $m b C D$ : a minimal logic with axioms $b c 1$ and $b d 1$. The definitions of syntactical and semantic consequence in $m b C D$ are analogous to those of $m b C$. Since $m b C D$ is both paracomplete

Manuscrito - Rev. Int. Fil., Campinas, v. 38, n.2, pp.155-184, jul.-ago. 2015. 
and paraconsistent in the sense that it permits valuations such that $v(A \vee$ $\neg A)=0$ and $v(A \wedge \neg A)=1$, it may express a scenario such that there is no evidence at all. The connective $\circ$ is still a consistent operator in the sense that $\circ A, A$, and $\neg A$ cannot hold together, but it can also be understood as a classicality operator, since $\circ A$ recovers classical truth conditions with respect to $A$.

It can be easily checked by the reader that monotonicity, the deduction theorem, and its converse (properties (2), (3) and (4) above) hold in $m b C D$, and that the classical negation is also definable in $m b C D$. A completeness proof for $m b C D$ is similar to the proof for $m b C$ presented in Carnielli et al. (2007) pp. 38-40. We just need to drop clauses (iv) and (v) of lemma 59, substituting them by the following:

$\circ \mathrm{B} \in \Delta$ implies $[\mathrm{B} \in \Delta$ iff $\neg \mathrm{B} \notin \Delta]$.

We now return to the intuitive interpretation of $\neg$ and $\circ$. Let us first consider the circumstance that only non-conclusive evidence is available, that is, that the truth value of $A$ has not been established yet. In this case $v(\circ A)=0$, and we have the following four possible scenarios:

A1.

There is evidence that $\mathrm{A}$ is the case;

$\mathrm{v}(\mathrm{A})=1$

There is evidence that $\mathrm{A}$ is not the case. $\quad \mathrm{v}(\neg \mathrm{A})=1$

A2.

There is evidence that $\mathrm{A}$ is the case;

$\mathrm{v}(\mathrm{A})=1$

There is no evidence that $\mathrm{A}$ is not the case. $\quad \mathrm{v}(\neg \mathrm{A})=0$ A3.

There is no evidence that $\mathrm{A}$ is the case; $\quad \mathrm{v}(\mathrm{A})=0$

There is evidence that $\mathrm{A}$ is not the case. $\quad \mathrm{v}(\neg \mathrm{A})=1$ A4.

There is no evidence that $\mathrm{A}$ is the case; $\quad \mathrm{v}(\mathrm{A})=0$ 
There is no evidence that $\mathrm{A}$ is not the case. $\quad \mathrm{v}(\neg \mathrm{A})=0$

A further step in our analysis is to consider circumstances where evidence is conclusive, that is, when a proposition has been established as true and so $\mathrm{v}(\circ \mathrm{A})=1$. We have only two possible (classical) scenarios:

A2*. There is conclusive evidence that $A$ is the case.

A3*. There is conclusive evidence that $A$ is not the case.

When $\mathrm{v}(\mathrm{\circ A})$ turns out to be 1 , we get scenarios A2* and A3*, respectively. Notice that $\mathrm{v}(\circ \mathrm{A})=1, \mathrm{v}(\mathrm{A})=1$, and $\mathrm{v}(\neg \mathrm{A})=1$ is not possible. Firstly, because once the truth of $A$ is conclusively established, any evidence for $\neg A$ is cancelled (and vice-versa), and secondly, because if $\circ A, A$ and $\neg A$ hold together, by $b c 1$ triviality obtains. We may have conflicting evidence, but not conflicting truth values. A remarkable feature of the above intuitive interpretation is that the simultaneous truth of a contradictory pair of propositions is not allowed, on pain of triviality. Under this interpretation, the system is not neutral with respect to true contradictions. On the other hand, $\mathrm{v}(\circ A)=0, \mathrm{v}(A)=0$, and $\mathrm{v}(\neg A)=0$ is also not possible, because it cannot be the case that the truth value of $A$ has been established but there is no evidence at all about $A$.

Let us return now to the validity of excluded middle in $m b C$. In $m b C$, the scenario $\mathrm{A} 4$, no evidence at all for both $A$ and $\neg A$ is excluded. Indeed, the semantic clause (iv) forbids that $A$ and $\neg A$ simultaneously receive the value 0 . Although we agree that the circumstance A4 in general should be allowed, there are some contexts of reasoning in which the validity of excluded middle may be justified.

Suppose a context in which a definite decision must be made in a finite amount of time. In such a context, it may be a methodological option to consider, by default, that $A$ receives the value 1 or that $\neg A$ receives the value 1 . In any case, the semantic value attributed to $A \vee$ $\neg A$ will always be 1 . Then, in order to describe such a context, axiom 10 , together with semantic clause (v), must hold. An example of a scenario in which the value 1 is attributed to $\neg A$ by default is a criminal 
investigation in which one starts by considering everyone (in some group of people) not guilty until there is proof to the contrary. Thus, whether or not excluded middle must be valid depends on the reasoning scenario we want to represent. We may understand such a situation as if there were evidence for not guilty for everyone in the group. It is worth noting, however, that the absence of any evidence does not by itself constitute evidence for $\neg A$. The attribution by default to evidence for $A$ (or $\neg A$ ) is a methodological decision external to the formal system.

There are some facts about the logic $m b C D$ that are worth mentioning.

1. The semantic clauses (i)-(iii) are exactly as in classical logic, and therefore all classical tautologies with $\wedge, \vee$, and $\rightarrow$ are valid. This is as expected, since $m b C D$ is an extension of positive classical logic.

2. An important feature of the semantics presented above is that both the negation and the consistency connectives are not truth-functional, that is, the semantic value attributed to $\neg A$ and $\circ A$ does not depend on the semantic value attributed to $A$. Since truth-functionality is a special case of compositionality, it is also remarkable that this semantics is not compositional in the sense that the semantic value of the whole expression is functionally determined by the semantic values of its parts and the way they are combined.

3. Clause (vi) expresses only a necessary condition for attributing the value 1 to $\circ A$ : we may have $v(A)=1$ and $v(\neg A)=0$ (or vice-versa) and $v(\circ A)=0$. We would like to call attention to the fact that according to clause (vi), $v(\circ A)=1$ implies that $v(A \wedge \neg A)=0$. Intuitively, if the truth-value of $A$ has been conclusively established, it cannot be that we still have evidence for both $A$ and $\neg A$. On the other hand, the converse does not hold: we may have $v(A \wedge \neg A)=0$ and $v(\circ A)=0$ when there is non-conclusive evidence for $A$ and no evidence at all for $\neg A$ (respectively $\neg A$ and $A$ ). Consistency and freedom from contradiction are independent.

Manuscrito - Rev. Int. Fil., Campinas, v. 38, n.2, pp.155-184, jul.-ago. 2015. 
4. It is easy to see that the law of explosion and disjunctive syllogism are not valid: the valuation $\mathrm{v}(A)=\mathrm{v}(\neg A)=1$ and $\mathrm{v}(B)=0$ makes both invalid. Intuitively, there may be evidence for $A$ and $\neg A$, hence for $A \vee$ $B$, but no evidence for $B$. In fact, disjunctive syllogism and the law of explosion are equivalent in the sense that, added to positive sentential logic (Ax.1-9 plus modus ponens), each one implies the other.

5. Modus tollens, as well as all versions of contraposition, does not hold. (10) $A \rightarrow B, \neg B \not \neg A$.

Is there an intuitive justification for the fact that modus tollens is not valid? The answer is affirmative. In classical logic, modus tollens is valid because the truth of $\neg B$ implies the falsity of $B$. Hence, in addition to truth being preserved by modus ponens, falsity is also preserved by modus tollens: given the truth of $A \rightarrow B$, if $B$ is false, $A$ is false too. This is not the case, however, under the interpretation we are proposing here. $\mathrm{v}(\neg B)=1$ means that we have some evidence that $B$ is not the case. This does not imply that we do not have simultaneous evidence for $B$, that is, $\mathrm{v}(\neg B)=1$ does not imply $\mathrm{v}(B)=0$. Suppose that we have evidence both for $A$ and $B$. In this case, $\mathrm{v}(A \rightarrow B)=1$ (notice that it is not required any kind of connection between the meanings of $A$ and $B$ in order to have $\mathrm{v}(A \rightarrow B)=1)$. Now, in order to see that modus tollens is not valid, make $\mathrm{v}(B)=\mathrm{v}(\neg B)=1$, and $\mathrm{v}(A)=1, \mathrm{v}(\neg A)=0$.

6. In $m b C$, as with the majority of logics of formal inconsistency, once the consistency of certain formulas is established, we get classical logic. A Derivability Adjustment Theorem (DAT), which recovers classical consequence once some information is added, holds for $m b C$ and can also be proved for $m b C D$ :

For all $\Gamma$ and for all $\mathrm{B}$ there is a $\Delta$ such that: $\Gamma \vDash_{\mathrm{C}} \mathrm{B}$ if and only if $\Gamma, \circ(\Delta) \vDash_{\mathrm{mbCD}} \mathrm{B}$, 
where $\circ(\Delta)=\{\circ \mathrm{A}: \mathrm{A} \in \Delta\}$ and $\vDash_{\mathrm{C}}$ means classical consequence. How a DAT works for $m b C D$ may be seen from the table below (all formulas in the left column are classically valid):

$$
\begin{aligned}
& \#_{\mathrm{mbCD}}(A \rightarrow B) \rightarrow\left((A \rightarrow \neg B) \circ A, \circ B \vDash_{\mathrm{mbCD}}(A \rightarrow B) \rightarrow((A \rightarrow\right. \\
& \rightarrow \neg A) \quad \neg B) \rightarrow \neg A) \\
& \|_{\mathrm{mbCD}} \neg \neg A \leftrightarrow A \quad \circ A, \circ \neg A \models_{\mathrm{mbCD}} \neg \neg A \leftrightarrow A \\
& \|_{\mathrm{mbCD}}(A \rightarrow \neg A) \rightarrow \neg A \quad \circ A \vDash_{\mathrm{mbCD}}(A \rightarrow \neg A) \rightarrow \neg A \\
& \#_{\mathrm{mbCD}}(\neg A \rightarrow A) \rightarrow A \quad \circ A \vDash_{\mathrm{mbCD}}(\neg A \rightarrow A) \rightarrow A \\
& \#_{\mathrm{mbCD}}(\mathrm{B} \rightarrow \mathrm{A}) \leftrightarrow\left(\neg \mathrm{A} \rightarrow \circ A, \circ B \vDash_{\mathrm{mbCD}}(\mathrm{B} \rightarrow \mathrm{A}) \leftrightarrow(\neg \mathrm{A} \rightarrow\right. \\
& \neg \mathrm{B}) \quad \neg \mathrm{B}) \\
& \#_{\mathrm{mbCD}}(\neg \mathrm{B} \rightarrow \mathrm{A}) \leftrightarrow\left(\neg \mathrm{A} \rightarrow \circ A, \circ B \vDash_{\mathrm{mbCD}}(\neg \mathrm{B} \rightarrow \mathrm{A}) \leftrightarrow(\neg \mathrm{A}\right. \\
& \text { B) } \rightarrow \text { B) }
\end{aligned}
$$

7. Finally, $m b C D$ is suitable for expressing the central idea of the intuitive interpretation of paraconsistent negation we have presented here, namely, that a contradiction $A \wedge \neg A$ means that there is conflicting evidence about $A$. Furthermore, since classical logic may be recovered within $m b C D, m b C D$ is a formal system able to deal simultaneously with preservation of evidence and preservation of truth.

\section{EPISTEMOLOGICAL CONTRADICTION AND THE NATURE OF LOGIC}


In this section we want to show that a formal system with a normative character designed to deal with epistemological contradictions finds its place in the very nature of logic. We start by calling attention to the fact that there is a perennial philosophical question about the nature of logic, namely, whether the main character of logic is epistemological, ontological, or linguistic. We emphasize the epistemological character of intuitionistic logic, which is in clear opposition to the realist (and, we claim, ontological) view of logic found in Frege's works. We argued in section 2 that at least some contradictions that appear in scientific theories are epistemological. Our main argument here depends on the duality between the rejection of explosion and the rejection of excluded middle, both of which may be motivated by epistemological reasons. From this perspective, the rejection of excluded middle does not mean that both $A$ and $\neg A$ may be false. Dually, the rejection of explosion does not mean that both $A$ and $\neg A$ may be true.

In building formal systems we deal with several logical principles, and it may justly be asked what these are principles about. This is a central issue in philosophy of logic. Here we follow Popper (1963, pp. 206ff), who presents the problem in a very clear way. The question is whether the rules of logic are:

(I.a) laws of thought in the sense that they describe how we actually think;

(I.b) laws of thought in the sense that they are normative laws, i.e., laws that tell us how we should think;

(II) the most general laws of nature, i.e., laws that apply to any kind of object;

(III) laws of certain descriptive languages.

We thus have three basic options, which are not mutually exclusive: the laws of logic have (I) epistemological, (II) ontological, or (III) linguistic character. With respect to (I), they may be (I.a) or descriptive (I.b) normative. Let us illustrate the issue with some examples.

Aristotle, defending the principle of non-contradiction, makes it clear that it is a principle about reality, "the most certain principle of all 
things" (Metaphysics 1005b11). Worth mentioning also is the well-known passage, "the same attribute cannot at the same time belong and not belong to the same subject in the same respect" (Metaphysics 1005b19-21), which is a claim about objects and their properties.

On the other hand, a very illustrative example of the epistemological side of logic can be found in the so-called logic of PortRoyal, where we read:

Logic is the art of conducting reasoning well in knowing things, as much to instruct ourselves about them as to instruct others.

This art consists in reflections that have been made on the four principal operations of mind: conceiving, judging, reasoning, and ordering.

(...) [T] his art does not consist in finding the means to perform these operations, since nature alone furnishes them in giving us reason, but in reflecting on what nature makes us do, which serves three purposes.

The first is to assure us that we are using reason well ...

The second is to reveal and explain more easily the errors or defects that can occur in mental operations.

The third purpose is to make us better acquainted with the nature of the mind by reflecting on its actions. (Arnauld, A. \& Nicole, 1996, p. 23)

Logic is conceived as having a normative character. So far so good. But logic is also conceived as a tool for analyzing mental processes of reasoning. This analysis, when further extended by different approaches to logical consequence, as is now done by some non-classical logics, shows that there can be different standards of correct reasoning in different situations. This aspect of logic, however, has been relegated to secondary status by Frege's attack on psychologism. Frege wanted to eliminate everything subjective from logic. For Frege, laws of logic cannot be obtained from concrete reasoning practices. Basically, his argument is the following. From the assumption that truth is not relative, it follows that the basic criterion for an inference to be correct, namely, the preservation of truth, should be the same for everyone. When different people make different inferences, we must have a criterion for deciding which one is correct. Combined with Frege's well-known 
Platonism, the result is a conception of logic that emphasizes the ontological (and realist) aspect of classical logic.

Our conception of the laws of logic is necessarily decisive for our treatment of the science of logic, and that conception in turn is connected with our understanding of the word 'true'. It will be granted by all at the outset that the laws of logic ought to be guiding principles for thought in the attainment of truth, yet this is only too easily forgotten, and here what is fatal is the double meaning of the word 'law'. In one sense a law asserts what is; in the other it prescribes what ought to be. Only in the latter sense can the laws of logic be called 'laws of thought' (...) If being true is thus independent of being acknowledged by somebody or other, then the laws of truth are not psychological laws: they are boundary stones set in an eternal foundation, which our thought can overflow, but never displace (Frege 1893, (1964) p. 13).

(...) $[\mathrm{O}]$ ne can very well speak of laws of thought too. But there is an imminent danger here of mixing different things up. Perhaps the expression "law of thought" is interpreted by analogy with "law of nature" and the generalization of thinking as a mental occurrence is meant by it. A law of thought in this sense would be a psychological law. And so one might come to believe that logic deals with the mental process of thinking and the psychological laws in accordance with which it takes place. This would be a misunderstanding of the task of logic, for truth has not been given the place which is its due here (Frege 1918, (1997) p. 325).

For Frege, logic is normative, but in a secondary sense. Along with truths of arithmetic, the logical relations between propositions are already given, eternal. This is not surprising at all. Since he wanted to prove that arithmetic could be obtained from purely logical principles, truths of arithmetic would inherit, so to speak, the realistic character of the logical principles from which they were obtained. Logic thus has an ontological character; it is part of reality, as are mathematical objects.

It is very interesting to contrast Frege's realism with Brouwer's intuitionism, whose basic ideas can be found for the first time in his doctoral thesis, written at the very beginning of twentieth century. The approaches are quite opposed.

Manuscrito - Rev. Int. Fil., Campinas, v. 38, n.2, pp.155-184, jul.-ago. 2015. 
Mathematics can deal with no other matter than that which it has itself constructed. In the preceding pages it has been shown for the fundamental parts of mathematics how they can be built up from units of perception (Brouwer 1907, (1975) p. 51).

The words of your mathematical demonstration merely accompany a mathematical construction that is effected without words ...

While thus mathematics is independent of logic, logic does depend upon mathematics: in the first place intuitive logical reasoning is that special kind of mathematical reasoning which remains if, considering mathematical structures, one restricts oneself to relations of whole and part (Brouwer 1907, (1975) p. 73-74).

It is remarkable that Brouwer's doctoral thesis (1907) was written between the two above-quoted works by Frege (1893 and 1919). Brouwer, like Frege himself, is primarily interested in mathematics. For Brouwer, however, the truths of mathematics are not discovered but rather constructed. Mathematics is not a part of logic, as Frege wanted to prove. Quite the contrary, logic is abstracted from mathematics. It is, so to speak, a description of human reasoning in constructing correct mathematical proofs. Mathematics is a product of the human mind, mental constructions that do not depend on language or logic. The raw material for these constructions is the intuition of time (this is the meaning of the phrase 'built up from units of perception').

These ideas are reflected in intuitionistic logic, which was formalized by Heyting (1956). Excluded middle is rejected precisely because mathematical objects are considered mental constructions. Accordingly, to assert an instance of excluded middle related to an unsolved mathematical problem (for instance, Goldbach's conjecture), would be a commitment to a Platonic realm of abstract objects, an idea rejected by Brouwer and his followers.

With respect to the linguistic aspects of logic, we shall make just a few comments. According to one widespread opinion, a linguistic conception of logic prevailed during the last century. From this perspective, logic has to do above all with the structure and functioning of certain languages. Indeed, sometimes logic is defined as a mathematical study of formal languages. There is no consensus for this 
view, however, and it is likely that it is not prevalent today. ${ }^{6}$ Even though we cannot completely separate the linguistic from the epistemological aspects - i.e., separate language from thought -, we endorse the view that logic is primarily a theory about reality and thought, and that the linguistic aspect is secondary. ${ }^{7}$

At first sight, it might seem that Frege's conception according to which there is only one logic, that is, only one account of logical consequence, is correct. Indeed, for Frege, Russell, and Quine, the logic is classical logic. From a realist point of view, this fits well with the perspective of the empirical sciences: excluded middle and bivalence have a strong appeal. Ultimately, reality will decide between $A$ and not $A$, which is the same as deciding between the truth and falsity of $A$.

The identification of an intuitionistic notion of provability with truth was not successful. As is shown by Raatikainen (2004), in the works of Brouwer and Heyting we find some attempts to formulate an explanation of the notion of truth in terms of provability, but all of them produce counterintuitive results.

On the other hand, the basic intuitionistic argument that rejects a supersensible realm of abstract objects is philosophically motivated and it is notable that this argument usually seems rather convincing to students of philosophy. As Velleman \& Alexander (2002, pp. $91 \mathrm{ff}$ ) put it, realism seems to be compelling when we consider a proposition like every star has at least one planet orbiting it. However, when we pass from this example to Goldbach's conjecture, the situation changes quite a bit. In the former case, it is very reasonable to say that reality is one way or the other; but if we say with regard to the latter case that 'the world of

\footnotetext{
${ }^{6}$ A rejection of the linguistic conception of logic, and an argument that logic is above all a theory with ontological and epistemological aspects, can be found in the Introduction to Chateaubriand (2001).

${ }^{7}$ Notice that this is in line with Chateaubriand's opinion, as manifested in Chateaubriand (2001, p. 16): "the fundamental character of logic is metaphysical, not linguistic. On the one hand I see it as an ontological theory that is part of a theory of the most general and universal features of reality; of being qua being, as Aristotle said. On the other hand I see it as an epistemological theory that is part of a general theory of knowledge."
}

Manuscrito - Rev. Int. Fil., Campinas, v. 38, n.2, pp.155-184, jul.-ago. 2015. 
mathematical numbers' is one way or the other, there is a question to be faced: where is this world?

What is the moral to be taken from this? That classical and intuitionistic logic are not talking about the same thing. The former is connected to reality through a realist notion of truth; the latter is not about truth, but rather about reasoning. In our view, assertability based on the intuitionistic notion of constructive proof is what is expressed by intuitionistic logic.

Now, one may ask what all of this has to do with logics of formal inconsistency and paraconsistent logics in general. The question concerning the nature of logic is a perennial problem of philosophy. We believe that it has no solution in the sense of some conclusive argument in defense of one or the other view. This is so, first, because logic is simultaneously about thought and reality, and, second, because different accounts of logical consequence may be more appropriate for expressing one view than another. We also claim that this is precisely the case with, on the one hand, classical logic and its ontological motivations and, on the other hand, with the epistemological approach of intuitionism and logics of formal inconsistency.

Let us take a look at the duality between intuitionistic and paraconsistent logics. In the latter, we may have two sentences $A$ and $\neg A$ with semantic value 1 ; non-contradiction (and explosion) does not hold. In the former, we may have two sentences $A$ and $\neg A$ (here ' $\neg$ ' is intuitionistic negation) with semantic value 0 ; excluded middle, the dual of non-contradiction, does not hold. If we stop to think about them for a moment, and put aside any realist bias, we see that we are facing two analogous situations.

At first sight, it really seems that an inference principle like modus tollens is valid whether we want it to be or not, that its validity is not a matter of any kind of choice or context whatever. This is indeed correct if we are talking about truth in the realist sense, a framework in which classical logic works well. However, we have seen above the reasons why modus tollens is not valid when we are reasoning in a context with a nonexplosive negation. Something analogous happens in intuitionistic logic.

Manuscrito - Rev. Int. Fil., Campinas, v. 38, n.2, pp.155-184, jul.-ago. 2015. 
Once we have endorsed a constructive notion of proof, we cannot carry out a proof by cases using excluded middle because the result might not be a constructive proof. When we say that logics of formal inconsistency accept some contradictions without exploding the system, this does not mean that these contradictions are true. We may compare this with Kripke models for intuitionistic logic, where it can happen that a pair of sentences $A$ and $\neg A$ ( $\neg$ here is intuitionistic negation) receive the value 0 in some stage (i.e., possible world). Such a stage would be a refutation of excluded middle. This does not mean that $A$ and not $A$ are false, however, but rather that neither has been proved yet. Analogously, as suggested above, when two sentences $A$ and $\neg A$ receive 1 in $m b C D$, it means that we have evidence for both, not that both are simultaneously true.

The position defended here with respect to paraconsistency thus differs fundamentally from dialetheism. According to Graham Priest and his collaborators,

A dialetheia is a sentence, $A$, such that both it and its negation, $\neg \mathrm{A}$, are true (..) Dialetheism is the view that there are dialetheias. (...) dialetheism amounts to the claim that there are true contradictions (Priest \& Berto 2013, Introduction).

One of the motivations for dialetheism is indeed the semantic and set theoretic paradoxes. But Priest tries to extend these motivations to empirical phenomena:

[T] he paradoxes of self-reference are not the only examples of dialetheias that have been mooted. Other cases involve contradictions affecting concrete objects and the empirical world (Priest \& Berto 2013, sec. 3.3) [T] here are, if not conclusive, then at least plausible reasons for supposing that these [discrete temporal changes] may produce dialetheias. (Priest 2006, p. 159)

In addition to the problem of the nature of logic mentioned above, the question of whether or not there are real contradictions, facts, and/or events in contradiction with each other, is a perennial problem in 
philosophy. It seems to us that this problem has no perspective of a conclusive solution, given the state of science at the present time. We agree, however, that the thesis that there are real contradictions, a view endorsed by Hegel and, according to some interpreters, Heraclitus, has an important place in the history of philosophy. On the other hand, it is not a contentious issue that contradictions do appear in the process of acquiring knowledge and dealing with data. In taking contradictions epistemologically, we have tried to show here that an intuitive justification for logics of formal inconsistency, and also for paraconsistency, that is not committed to dialetheism is entirely feasible.

\section{FINAL REMARKS}

We hope we have been successful in showing that having available a logical formalism capable of dealing with contradictions may have good philosophical motivations, and is not the same thing as having some kind of sympathy for ontological contradictions. We still believe that trying to avoid contradictions is an indispensable criterion of rationality. In order to do this, however, we need a logic that does not collapse in the face of a pair of contradictory propositions. As we have seen, a contradiction may be taken as a provisional state, a kind of excess of information that should, at least in principle, be eliminated by means of further investigation.

Notice that the semantics presented here is not, strictly speaking, a 'semantics of evidence', for this would require a compositional approach, formally clarifying how evidence is propagated through conjunction, disjunction, implication, negation, and the consistency operator. What we propose here is an intuitive interpretation of the bivalued semantics that, we claim, fits well with the basic features a formal system capable of expressing not only preservation of truth, but also preservation of evidence.

There is still a lot of work to be done on logics of formal inconsistency. As has been shown in Carnielli et al. (2007) and in Carnielli \& Marcos (2002), several systems may be formulated, with very

Manuscrito - Rev. Int. Fil., Campinas, v. 38, n.2, pp.155-184, jul.-ago. 2015. 
subtle differences among them. The task of finding intuitively acceptable semantics for non-classical logics is indeed a major philosophical challenge. Aside from the technical problems of finding adequate semantics, and of proving metatheorems, there are still a lot of open questions related to the intuitive interpretation of these systems and to the philosophical concepts expressed by them. It is very likely that the intuitive interpretation we have presented here can be improved. However, the point we want to emphasize is that $m b C D$ is an economical and elegant formal system that has the resources to express the central features of non-dialetheist reading of paraconsistency.

\section{ACKNOWLEDGEMENTS}

The first author acknowledges the support of FAPESP (São Paulo Research Council) and CNPq, Brazil (The National Council for Scientific and Technological Development). The second author acknowledges support from the Universidade Federal de Minas Gerais (edital 12/2011) and Fundação de Amparo à Pesquisa do Estado de Minas Gerais (FAPEMIG research project 21308).

\section{REFERENCES}

Arnauld, A.; Nicole, P. Logic or the Art of Thinking. Cambridge University Press, 1996.

AristotLe. Metaphysics. The Complete Works of Aristotle, Oxford University Press, 1996.

Brouwer, L.E.J. "On the Foundations of Mathematics" 1907. Collected Works vol. I. (ed. A. Heyting), North-Holland Publishing Company, 1975.

CARnielli, W. "The Single-minded Pursuit of Consistency and its Weakness" in Studia Logica, v. 97, p. 81-100, 2011. 
Carnielli, W.; Coniglio, M.; Marcos, J. "Logics of Formal Inconsistency". Handbook of Philosophical Logic, vol. 14, pp.15107, (eds.: D. Gabbay; F. Guenthner). Springer, 2007.

Carnielli, W.; Marcos, J. "A Taxonomy of C-systems". Paraconsistency: the logical way to inconsistency, Proceedings of the Second World Congress on Paraconsistency. New York: Marcel Dekker, 2002.

Chateaubriand, O. Logical forms vol. 1. Campinas: UNICAMP-CLE, 2001.

DA Costa, N. "The philosophical import of paraconsistent logic". Journal of Non-Classical Logics, number 1, pp. 1-19, 1982.

Da Costa, N.; French, S. Science and Partial Truth. Oxford University Press, 2003.

Frege, G. The Basic Laws of Arithmetic, 1893. Transl. M. Furth. University of California Press, 1964.

. "The Thought", 1918. The Frege Reader. Oxford: Blackwell Publishers, 1997.

Heyting, A. Intuitionism: an Introduction. London: North-Holland Publishing Company, 1956.

Hunter, G. Metalogic. University of California Press, 1973.

NiCKLES, T. 'From Copernicus to Ptolemy: inconsistency and method' in Inconsistency in Science (Ed. J. Meheus). Dordrecht: Springer, 2002.

POPPER, K. Conjectures and Refutations, New York: Harper, 1963. 
PRIEST, G. In Contradiction. Oxford University Press, 2006.

Priest, G.; Berto, F. "Dialetheism" in Stanford Encyclopedia of Philosophy.

http://plato.stanford.edu/archives/sum2013/entries/dialetheism/ , 2013.

RaAtikainen, P. "Conceptions of Truth in Intuitionism”. History and Philosophy of Logic, 25: 131-145, 2004.

RobBIn, J.W. Mathematical Logic: A First Course. New York: Dover, 1997.

Velleman, D.J.; Alexander George, A. Philosophies of Mathematics. Oxford: Blackwell Publishers, 2002. 